\title{
Self-rated eyesight and handgrip strength in older adults
}

Lee Smith ${ }^{*}$, Peter Allen ${ }^{2}$, Shahina Pardhan ${ }^{3}$, Trish Gorely ${ }^{4}$, Igor Grabovac ${ }^{5}$, Annetta Smith ${ }^{4}$, Guillermo F. López-Sánchez ${ }^{6}$, Lin Yang ${ }^{7}$, Sarah E. Jackson $^{8}$

1. The Cambridge Centre for Sport and Exercise Sciences, Anglia Ruskin University, Cambridge, UK

2. Department of Vision and Hearing Sciences \& Vision and Eye Research Unit, Anglia Ruskin University, Cambridge, UK

3. Vision and Eye Research Unit (VERU), Postgraduate Medical Institute, Anglia Ruskin University, Cambridge

4. Department of Nursing, University of the Highlands and Islands, Inverness, UK

5. Department of Social and Preventive Medicine, Centre for Public Health, Medical University of Vienna, Vienna, Austria

6. Faculty of Sport Sciences, University of Murcia, Murcia, Spain

7. Department of Epidemiology, Centre for Public Health, Medical University of Vienna, Vienna, Austria

8. Department of Behavioural Science and Health, University College London, London, UK

*Corresponding author: Dr Lee Smith; The Cambridge Centre for Sport and Exercise Sciences, Anglia Ruskin University, Cambridge, UK, CB1 1PT; lee.smith@anglia.ac.uk 


\title{
Self-rated eyesight and handgrip strength in older adults
}

\begin{abstract}
Background: The aim of this study was to investigate the association between self-rated eyesight and handgrip strength in a large, representative population of older adults.

Methods: Data were from 7,433 older adults ( $\geq 52 \mathrm{y})$ participating in the English Longitudinal Study of Ageing. We used linear regression to analyse the association between self-rated eyesight and handgrip strength cross-sectionally in 2004/05, and longitudinally over four-year follow-up, adjusting for a range of socio-demographic and health-related variables.
\end{abstract}

Results: In cross-sectional and prospective models, poor eyesight was strongly associated with lower handgrip strength after adjustment for age, sex, ethnicity, socioeconomic status and BMI (crosssectional $\mathrm{B}=-1.39 \mathrm{~kg}, 95 \% \mathrm{CI}-1.84$ to $-0.94, p<0.001$, prospective $\mathrm{B}=-0.68 \mathrm{~kg}, 95 \% \mathrm{CI}-1.14$ to $0.22, p=0.004)$. The association was attenuated but remained statistically significant when health behaviours were included in the model (cross-sectional $\mathrm{B}=-0.93 \mathrm{~kg}, 95 \% \mathrm{CI}-1.42$ to $-0.44, p<0.001$, prospective $\mathrm{B}=-0.50,95 \% \mathrm{CI}-0.99$ to $-0.02, p=0.044)$.

Conclusions: Older adults in England with poor self-rated eyesight have lower levels of physical function compared with those with good eyesight. This association can be predominantly explained by differences in age, sex, ethnicity, socioeconomic status, BMI, and health behaviours, as well as chronic conditions, disability and depression.

Key Words: Vision, Handgrip strength, Older adults, ELSA, Retinopathy. 


\section{INTRODUCTION}

Population ageing has resulted in a rise in the number of people living with visual impairment. In the UK, approximately two million people are blind or partially sighted, with particularly high prevalence among older people (increasing from $10.8 \%$ in those aged $75-79$ to $53.1 \%$ in those aged $\geq 90$ years) [1]. Evidence suggests that older people with visual impairment are at increased risk of a variety of adverse outcomes including depression [2], cognitive decline [3] and poor general health [4]. This study examines the relationship between visual impairment and another important aspect of ageing: physical function.

As adults age, a decline in physical function is generally observed [5-7]. Reduced physical performance increases the risk of falls, level of dependency, health care use, admissions to residential care and even mortality $[6,8,9]$. Everyday tasks such as climbing stairs require functioning close to maximum capacity in some older adults, so any decline increases the risk of becoming dependent on a carer or institutionalized [10].

Poor physical function may be particularly prevalent among older adults with reduced vision. Previous research has shown that levels of physical activity - a key predictor of physical function [11] - are lower across the lifespan in people with poor vision than their same-age counterparts [12,13]. Few studies have explicitly tested the association between visual impairment and physical performance in older age, but those that have indicate decrements among those with poor vision. One study of middleaged women ( $n=483$, age $42-56$ years) observed poorer performance on a battery of tests (including a 40-foot timed walk, timed stair climb and forward reach) in women with impaired vision over 10-year follow-up [14]. Similarly, a study of 6,112 women aged 69 and older reported an association between visual impairment and functional decline [3]. Another study of 1,504 older adults (72-92 years) found that vision loss was associated with a decline in mobility performance, measured by walking speed, 
number of bumps and number of orientation errors on a 32.8m obstacle course [15]. Similarly, vision loss was associated with poorer mobility (timed walk, chair rise, stair climb) and reduced ability to perform daily activities in a study of 2,520 65-84 year olds [16].

While these studies provide a useful starting point for research in this area, there is a need for further research for several reasons. Firstly, all of these studies were conducted in the US. While samples were recruited from both urban and rural areas, increasing the likelihood that results may be representative for people living in the US, it is not clear how generalisable such findings are for other developed countries. Further research in other developed countries is thus needed. Second, there is little understanding of the mechanisms underlying low levels of physical functioning among older people with visual impairment. Identifying why older adults with poor vision tend to have lower levels of physical function could help to inform targeted interventions. Finally, tests of physical function in these studies were fairly resource and time-intensive. There is a need for research using a practical measure that can be easily implemented into a healthcare setting.

The present study aimed to address these gaps in the literature by investigating the association between self-rated eyesight (a proxy measure for visual impairment) and handgrip strength (a marker of physical function) in a large, representative population of older adults. There is a growing body of evidence to show that handgrip strength is a valid measure of physical function and is a non-invasive and "quick" measure of physical health used in research and clinical settings [17-20]. Importantly, it has been suggested that a handheld dynamometer (to measure grip strength) could be a useful instrument in geriatric practice to identify those at risk of disability [21]. Using data collected over a four-year period as part of the English Longitudinal Study of Ageing (ELSA), we analysed crosssectional and prospective associations between poor self-rated eyesight and handgrip strength, with adjustment for a broad range of relevant socio-demographic and health-related covariates. 


\title{
PATIENTS, MATERIALS AND METHODS
}

\section{Study population}

ELSA is a population-representative longitudinal panel study of men and women aged $\geq 50$ years living in England [22]. Participants take part in biennial assessments, in which they complete a computer assisted personal interview and self-completion questionnaires, with a nurse visit in alternate (even) waves to collect objective measures of health status, including handgrip strength. This study uses data from Wave 2 (2004/05; the first wave to include a nurse visit) and Wave 4 (2008/09; selected over more distal waves in order to maximise the available sample for analysis). Of the 9,432 individuals who took part in Wave 2 of ELSA, 7,666 (81.3\%) completed the nurse visit. We excluded 233 participants with missing data on self-rated eyesight or handgrip strength in Wave 2, leaving a final sample for analysis of 7,433 men and women. Follow-up data were available for 4,895 (65.9\%) participants. Ethical approval was obtained from the London Multi- Centre Research Ethics Committee. All participants gave full informed consent to participate in the study.

\author{
Measures \\ $\underline{\text { Exposure: self-rated eyesight }}$ \\ The measure of self-rated eyesight was a single-item rating, which asked "Is your eyesight (using \\ glasses or corrective lenses; if you use them) excellent/very good/good/fair/or poor?" Spontaneous \\ responses of legally or registered blind were recorded. We dichotomised responses, defining visual \\ impairment as blindness or fair or poor self-rated eyesight (hereafter referred to as "poor vision").
}




\section{Outcome: handgrip strength}

Handgrip strength (kg) was assessed using the Smedley hand-held dynamometer (Stoelting Co, IL, USA). Participants were required to hold the device at a right angle to their body and exert maximum force for a couple of seconds when instructed. Successive trials were alternated between dominant and non-dominant hands. Three measurements were taken from each hand and the highest reading used in the present analysis.

\section{$\underline{\text { Covariates }}$}

All covariates were selected a priori. Demographic information included age, sex, ethnicity (white vs. non-white) and household non-pension wealth (a sensitive indicator of socioeconomic status in this age group) [23]. Body mass index (BMI) was calculated as weight in $\mathrm{kg}$ divided by the square of height in metres, based on objective measures taken by trained research nurses. Health behaviours were assessed with questions that asked participants about their current smoking status (smoker vs. non-smoker), frequency of alcohol intake (categorised as: never/rarely [never - once or twice a year], regularly [once every couple of months - twice a week], or frequently [3 days a week - almost every day]), and level of physical activity (categorised as: inactive [no moderate/vigorous activity on a weekly basis], moderate activity at least once a week, and vigorous activity at least once a week) [24]. Participants reported the presence or absence of limiting long-standing illness, defined as any longstanding illness, disability, or infirmity that limits activities in any way. Disability was assessed based on participants' responses to questions on perceived difficulty performing basic activities of daily living (e.g. difficulty dressing, including putting on shoes and socks) [25]. Finally, depressive symptoms were assessed using the eight-item Centre for Epidemiological Studies Depression Scale, a scale validated for use in older adults [26]. 


\section{Statistical analysis}

Analyses were conducted using SPSS version 24, with survey weights applied to account for sampling probabilities and non-response. For cross-sectional analyses, the weights accounted for the differential probability of being included in Wave 2 of ELSA and for non-participation in the nurse visit in which handgrip strength was measured. For prospective analyses, we applied a longitudinal weight that accounted for nonresponse at Wave 4 based on the sample who participated in baseline.

Associations between self-rated eyesight and covariates were assessed using one-way independent analysis of variance for continuous variables and chi-square tests for categorical variables. We then constructed a series of linear regression models to analyse cross-sectional and prospective associations between self-rated eyesight and handgrip strength. The models sequentially adjusted for age and sex (Model 1), other socio-demographic characteristics (ethnicity, wealth; Model 2), BMI (Model 3), health behaviours (physical activity, smoking, alcohol; Model 4), health status (limiting long-standing illness, disability; Model 5), and depressive symptoms (Model 6). Prospective models were additionally adjusted for baseline handgrip strength.

\section{RESULTS}

Our sample of 7,433 participants ranged in age from 52 to 99 years (mean 66.80, SD 10.47). Poor vision was reported by $13.5 \%$ of participants. Table 1 summarises sample characteristics in relation to self-rated eyesight. On average, participants with poor eyesight were significantly older compared with the group with good eyesight, and a higher proportion were female, non-white and from the lowest quintiles of wealth. Their BMI was significantly lower, but they were more likely to be inactive. They were more likely to smoke but less likely to drink alcohol frequently. Prevalence of limiting 
long-standing illness and disability were higher among people with poor eyesight, and they reported more depressive symptoms.

Associations between self-rated eyesight and handgrip strength are presented in Table 2. Crosssectionally, the mean age and sex-adjusted handgrip strength was $27.7 \mathrm{~kg}$ (SE 0.21) in older adults with poor eyesight vs. $29.8 \mathrm{~kg}$ (SE 0.08) in those with good eyesight, such values are similar to normative values [27]; a difference of $-2.04 \mathrm{~kg}(95 \% \mathrm{CI}-2.49$ to $-1.58, p<0.001)$. Poor eyesight remained strongly associated with lower handgrip strength after further adjustment for ethnicity, wealth and BMI (adjusted mean difference $-1.39 \mathrm{~kg}, 95 \% \mathrm{CI}-1.84$ to $-0.94, p<0.001$ ). The association was attenuated when health behaviours (adjusted mean difference $-0.93 \mathrm{~kg}, 95 \%$ CI -1.42 to -0.44 , $p<0.001$ ) and then limiting long-standing illness and disability were included in the model (adjusted mean difference $-0.50 \mathrm{~kg}, 95 \% \mathrm{CI}-0.99$ to $-0.02, p=0.044)$, and became non-significant after additional adjustment for depressive symptoms (adjusted mean difference $-0.35 \mathrm{~kg}, 95 \% \mathrm{CI}-0.84$ to $0.15, p=0.167)$. Overall, the covariates explained $82.8 \%$ of the cross-sectional age and sex-adjusted association between self-rated eyesight and handgrip strength.

A similar pattern of results was observed in the prospective models. The mean age and sex-adjusted handgrip strength was 28.7 (SE 0.10) in older adults with poor eyesight vs. 26.7 (SE 0.28) in those with good eyesight. Significantly lower handgrip strength was observed in the group with poor vision after adjustment for age, sex, ethnicity, wealth and BMI (adjusted mean difference $-0.68 \mathrm{~kg}, 95 \% \mathrm{CI}$ -1.14 to $-0.22, p=0.004$ ) and health behaviours (adjusted mean difference $-0.50 \mathrm{~kg}, 95 \% \mathrm{CI}-0.99$ to $0.02, p=0.044)$. A marginal but non-significant association remained after adjustment for limiting long-standing illness, disability and depressive symptoms (adjusted mean difference $-0.44 \mathrm{~kg}, 95 \%$ CI -0.94 to $0.06, p=0.084)$. Overall, the covariates explained $40.5 \%$ of the prospective age and sexadjusted association between self-rated eyesight and handgrip strength. 


\section{DISCUSSION}

In this large, representative sample, we found that older adults with poor self-rated eyesight had weaker grip strength than age-matched counterparts, both cross-sectionally and over four-year followup. Sequential adjustment for sociodemographic characteristics, health behaviours, the presence of chronic conditions, disability and depression saw a reduction in the strength of this association, with these variables accounting for $82.8 \%$ of the cross-sectional association and $40.5 \%$ of the prospective association. However, even after taking into account the aforementioned variables there remained a marginal difference in handgrip strength between older adults with poor and good self-rated eyesight.

The present findings are in line with previous studies that have shown that older people with visual impairment have worse levels of physical functioning than those with normal vision, using less practical measures of functioning (e.g. an obstacle course or a battery of fitness tests) and smaller samples [3,14-16]. The present study adds to this literature by showing that people with visual impairment have slightly worse levels of handgrip strength which can consequently be associated with lower physical functioning. Clinicians may want to consider using handgrip strength, a practical measure for physical functioning, in clinical practice.

An important contribution of this study to the existing literature is the insight offered into why older people with poor vision have impaired physical function. In both cross-sectional and prospective models, the basic age and sex-adjusted model was partially explained by differences in sociodemographic characteristics between the groups with poor and good eyesight: ethnicity and wealth (an index of socioeconomic status). These variables have previously been identified as important determinants of visual impairment [28] and physical function [29-30]. 
Health behaviours (including smoking, alcohol and physical activity) were found to play an important role. These variables have also been shown to be associated with general strength in the general population $[11,31,32]$. Interventions that target a reduction in smoking and an increase in physical activity will likely increase handgrip strength and thus physical function in this population. Drawing visually impaired smokers' attention to the potential impact of smoking on their physical function may provide increased motivation for them to quit. Healthcare professionals may wish to raise this issue with older patients with poor eyesight and provide advice on aids to cessation known to increase success rates (e.g. varenicline, nicotine replacement therapy, behavioural support) [33-35]. When intervening to promote physical function in those with poor self-rated eyesight it may be possible to increase physical activity by overcoming common exercise barriers in this population, such as transport and lack of accessible exercise equipment [36-37] and promoting exercises that can be done at home.

Health status (limiting long-standing illness and disability) also appeared to be an important mediator of the association between visual impairment and handgrip strength. With visually impaired older adults at increased risk of chronic health conditions [4], many of which are caused or exacerbated by inactivity and smoking, this provides even more reason to develop targeted interventions to improve these health behaviours among the visually impaired population.

Finally, depression was also identified as a variable partly driving the association between self-rated eyesight and handgrip strength. Those who are visually impaired are at a high risk of depression [2] which may lead to low levels of physical activity and reduced physical function. Healthcare professionals should maintain awareness of this link and respond promptly to signs of depression in this population. Interventions that target an improvement in depressive symptoms in those with visual impairment are likely to also have benefits for physical function. 
It is important to note that some of the differences observed in the present study in handgrip strength between those with poor vs. good eyesight was relatively low $(-0.5 \mathrm{~kg})$. However, it has been shown that in older adults with a weak grip strength at baseline there is an average yearly decline in grip strength of $0.5 \mathrm{~kg}$ to $0.8 \mathrm{~kg}$ [38]. Other studies have shown a decline as low as $0.3 \mathrm{~kg}$ [39]. Therefore, to observe a difference of $-0.5 \mathrm{~kg}$ in older adults with visual impairment compared with the general population is thus likely clinically meaningful. Next, on some classic analogue handgrip strength dynamometer such a small difference is not scalable. Those measuring handgrip strength in clinic, for example, should be encouraged to use the more precise digital measures of grip strength, such as the Jamar hydraulic dynamometer (Sammons Preston Rolyan, USA), which has a precision of $0.5 \mathrm{~kg}$ and a range of zero to $90 \mathrm{~kg}$.

Strengths of the present study include the large, representative sample, prospective design and inclusion of a range of relevant covariates in order to provide insight into the mechanisms underlying differences in grip strength between older people with poor vs. good eyesight. However, findings from the present study must be interpreted in light of its limitations. Visual impairment was determined by self-report of poor eyesight or blindness, introducing scope for bias. There is a need to replicate our findings using a more objective assessment of vision loss. Around a third of our baseline sample were lost to follow-up, and compared with the full sample, those who provided follow-up data were on average significantly younger and wealthier, had higher handgrip strength, and a higher proportion rated their eyesight as good. It is therefore possible that our prospective results underestimate the association between self-rated eyesight and decline in handgrip strength. Finally, it should be noted that the present data was collected in Wave 2 (2004/05) and Wave 4 (2008/09) of the ELSA study meaning the data analysed in this paper is now 10 to 15 years old. It may be possible that if recent data were analysed different findings may be observed. However, while this is often the case when analysing behavioural variables there is no plausible explanation why the association would be different when considering the analysed physical health variables in this study. Nevertheless, analyses using more recent data are now required to either confirm or refute the present findings. 
In conclusion, the present results indicate that older adults with poor vision have weaker handgrip strength than those with good vision. This association can be predominantly explained by differences in age, sex, ethnicity, SES, and health behaviours, as well as chronic conditions, disability and depression. Targeted interventions to improve health behaviours could improve physical function and reduce the burden of poor health and dependence in the visually impaired population in later life.

Declarations of interest: All authors (LS, PA, SP, TG, IG, AS, GFLS, LY, SEJ) declare that there is no conflict of interest.

Funding: Dr GFLS is funded by the Seneca Foundation-Agency for Science and Technology of the Region of Murcia, Spain. 20390/PD/17.

Ethical standards statement: All procedures followed were in accordance with the ethical standards of the responsible committee on human experimentation (institutional and national) and with the Helsinki Declaration of 1975, as revised in 2008.

Statement of informed consent: Informed consent was obtained from all patients for being included in the study.

\section{REFERENCES}

1. Royal National Institute of Blind People- RNIB. Future sight loss UK 1. Supporting people with sight loss. 2014. [cited 2019 May 15]. Available from: https://www.rnib.org.uk/knowledge-and-researchhub/research-reports/general-research/future-sight-loss-uk-1 
2. Choi HG, Lee MJ, Lee SM. Visual impairment and risk of depression: A longitudinal follow-up study using a national sample cohort. Sci Rep. 2018;8(1):1-8.

3. Lin MY, Gutierrez PR, Stone KL, et al. Vision Impairment and Combined Vision and Hearing Impairment Predict Cognitive and Functional Decline in Older Women. J Am Geriatr Soc. 2004;52(12):1996-2002.

4. Crews JE, Campbell VA. Vision Impairment and Hearing Loss Among Community-Dwelling Older Americans: Implications for Health and Functioning. Am J Public Health. 2004;94(5):823-829.

5. Veronese N, Stubbs B, Trevisan C, et al. Poor Physical Performance Predicts Future Onset of Depression in Elderly People: Progetto Veneto Anziani Longitudinal Study. Phys Ther. 2017;97(6):659-668.

6. Veronese N, Stubbs B, Fontana L, et al. A Comparison of Objective Physical Performance Tests and Future Mortality in the Elderly People. J Gerontol A Biol Sci Med Sci. 2017;72(3):362-368.

7. Veronese N, Stubbs B, Trevisan C, et al. What physical performance measures predict incident cognitive decline among intact older adults? A 4.4year follow up study. Exp Gerontol. 2016;81:110-118.

8. Gill TM, Kurland B. The burden and patterns of disability in activities of daily living among communityliving older persons. J Gerontol A Biol Sci Med Sci. 2003;58(1):70-75.

9. Freedman VA, Martin LG, Schoeni RF. Recent trends in disability and functioning among older adults in the United States: a systematic review. JAMA. 2002;288(24):3137-3146.

10. Rikli RE, Jones CJ. Development and Validation of a Functional Fitness Test for Community-Residing Older Adults. J Aging Phys Act. 1999;7(2):129-161.

11. Paterson DH, Warburton DE. Physical activity and functional limitations in older adults: a systematic review related to Canada's Physical Activity Guidelines. Int J Behav Nutr Phys Act. 2010;7(1):1-22. 
12. Smith L, Timmis MA, Pardhan S, et al. Physical inactivity in relation to self-rated eyesight: cross-sectional analysis from the English Longitudinal Study of Ageing. BMJ Open Ophthalmol. 2017;1(1):e000046.

13. Safeek RH, Hall KS, Lobelo F, et al. Low Levels of Physical Activity Among Older Persons Living with HIV/AIDS Are Associated with Poor Physical Function. AIDS Res Hum Retroviruses. 2018;34(11):929-935.

14. Chandrasekaran N, Harlow S, Moroi S, et al. Visual Impairment at Baseline is Associated with Future Poor Physical Functioning Among Middle-Aged Women: The Study of Women's Health Across the Nation, Michigan site. Maturitas. 2017;96:33-38.

15. Turano KA, Broman AT, Bandeen-Roche K, et al. Association of Visual Field Loss and Mobility Performance in Older Adults: Salisbury Eye Evaluation Study. Optom Vis Sci. 2004;81(5):298-307.

16. West SK, Rubin GS, Broman AT, et al. How Does Visual Impairment Affect Performance on Tasks of Everyday Life?: The SEE Project. Arch Ophthalmol. 2002;120(6):774-780.

17. Onder G, Penninx BWJH, Ferrucci L, et al. Measures of physical performance and risk for progressive and catastrophic disability: results from the Women's Health and Aging Study. J Gerontol A Biol Sci Med Sci. 2005;60(1):74-79.

18. Giampaoli S, Ferrucci L, Cecchi F, et al. Hand-grip strength predicts incident disability in non-disabled older men. Age Ageing. 1999;28(3):283-288.

19. Yang L, Koyanagi A, Smith L, et al. Hand grip strength and cognitive function among elderly cancer survivors. PLOS ONE. 2018;13(6):e0197909.

20. Smith L, White S, Stubbs B, et al. Depressive symptoms, handgrip strength, and weight status in US older adults. J Affect Disord. 2018;238:305-310. 
21. Taekema DG, Gussekloo J, Maier AB, Westendorp RGJ, de Craen AJM. Handgrip strength as a predictor of functional, psychological and social health. A prospective population-based study among the oldest old. Age Ageing. 2010;39(3):331-337.

22. Steptoe A, Breeze E, Banks J, Nazroo J. Cohort profile: the English Longitudinal Study of Ageing. Int J Epidemiol. 2013;42(6):1640-1648.

23. Banks J, Karlsen S, Oldfield Z. Socio-economic position. 2003 [cited 2019 May 15]; Available from: http://discovery.ucl.ac.uk/15366/1/15366.pdf

24. Hamer M, Molloy GJ, de Oliveira C, Demakakos P. Leisure time physical activity, risk of depressive symptoms, and inflammatory mediators: the English Longitudinal Study of Ageing.

Psychoneuroendocrinology. 2009;34(7):1050-1055.

25. Katz S, Downs TD, Cash HR, Grotz RC. Progress in development of the index of ADL. The Gerontologist. 1970;10(1):20-30.

26. Steffick DE. Documentation of affective functioning measures in the Health and Retirement Study. Ann Arbor, MI: University of Michigan; 2000.

27. Araújo Amaral C, Lameira Maciel Amaral T, Torres G, Monteiro R, Teixeira Leite Vasconcellos M, Crisóstomo Portela M. Hand grip strength: Reference values for adults and elderly people of Rio Branco, Acre, Brazil. PLoS One. 2019;14(1):e0211452. doi: 10.1371/journal.pone.0211452

28. Tielsch JM, Sommer A, Katz J, Quigley H, Ezrine S. Socioeconomic Status and Visual Impairment Among Urban Americans. Arch Ophthalmol. 1991;109(5):637-641. 
29. Kuh D, Bassey EJ, Butterworth S, Hardy R, Wadsworth MEJ. Grip Strength, Postural Control, and Functional Leg Power in a Representative Cohort of British Men and Women: Associations With Physical Activity, Health Status, and Socioeconomic Conditions. J Gerontol Ser A. 2005;60(2):224-231.

30. Guralnik JM, Butterworth S, Wadsworth MEJ, Kuh D. Childhood Socioeconomic Status Predicts Physical Functioning a Half Century Later. J Gerontol Ser A. 2006;61(7):694-701.

31. Baumgartner RN, Waters DL, Gallagher D, Morley JE, Garry PJ. Predictors of skeletal muscle mass in elderly men and women. Mech Ageing Dev. 1999;107(2):123-136.

32. Al-Obaidi S, Al-Sayegh N, Nadar M. Smoking impact on grip strength and fatigue resistance: implications for exercise and hand therapy practice. J Phys Act Health. 2014;11(5):1025-1031.

33. Hartmann-Boyce J, Chepkin SC, Ye W, Bullen C, Lancaster T. Nicotine replacement therapy versus control for smoking cessation. Cochrane Database Syst Rev. 2018;5:1-174.

34. Stead LF, Buitrago D, Preciado N, et al. Physician advice for smoking cessation. Cochrane Database Syst Rev. 2013;2(2):1-56.

35. Stead LF, Lancaster T. Combined pharmacotherapy and behavioural interventions for smoking cessation. Cochrane Database Syst Rev. 2012;10:CD008286.

36. Capella-McDonnall M. The Need for Health Promotion for Adults Who Are Visually Impaired. J Vis Impair Blind. 2007;101(3):133-145.

37. Phoenix C, Griffin M, Smith B. Physical activity among older people with sight loss: a qualitative research study to inform policy and practice. Public Health. 2015;129(2):124-130. 
38. Granic A, Davies K, Martin-Ruiz C, et al. Grip strength and inflammatory biomarker profiles in very old adults. Age and Ageing, 2017;46(6):976-982. doi: 10.1093/ageing/afx088

39. Granic A, Davies K, Jagger C, et al. Grip Strength Decline and Its Determinants in the Very Old: Longitudinal Findings from the Newcastle 85+ Study. PLoS One, 2016;11(9): e0163183. doi: 10.1371/journal.pone.0163183

Table 1 Sample characteristics at baseline in relation to self-rated eyesight

\begin{tabular}{|c|c|c|c|}
\hline & $\begin{array}{c}\text { Good } \\
(n=6433)^{1}\end{array}$ & $\begin{array}{c}\text { Poor } \\
(n=1000)\end{array}$ & $p$ \\
\hline Age (years), mean (SD) & $66.04(9.75)$ & $71.30(11.83)$ & $<0.001$ \\
\hline \multicolumn{4}{|l|}{ Sex } \\
\hline Men & 47.8 & 38.9 & $<0.001$ \\
\hline Women & 52.2 & 61.1 & - \\
\hline \multicolumn{4}{|l|}{ Ethnicity } \\
\hline White & 97.9 & 95.6 & $<0.001$ \\
\hline Non-white & 2.1 & 4.4 & - \\
\hline \multicolumn{4}{|l|}{ Wealth quintile } \\
\hline 1 (poorest) & 16.6 & 32.5 & $<0.001$ \\
\hline 2 & 18.9 & 25.1 & - \\
\hline 3 & 20.9 & 17.3 & - \\
\hline 4 & 21.4 & 14.2 & - \\
\hline 5 (richest) & 22.2 & 10.9 & - \\
\hline Body mass index (kg), mean (SD) & $26.44(7.88)$ & $24.71(10.23)$ & $<0.001$ \\
\hline \multicolumn{4}{|l|}{ Physical activity } \\
\hline Inactive & 21.1 & 46.5 & $<0.001$ \\
\hline Moderate at least once a week & 50.0 & 39.0 & \\
\hline Vigorous at least once a week & 28.9 & 14.6 & - \\
\hline \multicolumn{4}{|l|}{ Smoking status } \\
\hline Non-smoker & 84.6 & 80.3 & $<0.001$ \\
\hline Smoker & 15.4 & 19.7 & - \\
\hline
\end{tabular}

Alcohol intake 


\begin{tabular}{llcc} 
Never/rarely & 18.7 & 30.9 & $<0.001$ \\
\hline Regularly & 45.6 & 42.7 & \\
\hline Frequently & 35.7 & 26.5 & - \\
\hline $\begin{array}{l}\text { Limiting long-standing illness } \\
\text { Absent }\end{array}$ & 68.1 & 39.9 & $<0.001$ \\
\hline Present & 31.9 & 60.1 & - \\
\hline $\begin{array}{l}\text { Disability } \\
\text { Absent }\end{array}$ & 82.3 & 60.8 & $<0.001$ \\
\hline Present & 17.7 & 39.2 & - \\
\hline Depressive symptoms (0-8), mean (SD) & $1.41(1.83)$ & $2.55(2.28)$ & $<0.001$
\end{tabular}

${ }^{1}$ Unweighted sample sizes.

All figures are weighted for sampling probabilities and differential non-response.

Values are percentages unless otherwise stated.

$\mathrm{SD}=$ standard deviation . 
Table 2 Cross-sectional associations between self-rated eyesight and handgrip strength

\begin{tabular}{|c|c|c|c|c|c|c|}
\hline \multirow[b]{2}{*}{ Model } & \multicolumn{3}{|c|}{ Cross-sectional } & \multicolumn{3}{|c|}{ Prospective $^{1}$} \\
\hline & $\mathrm{B}^{2}[95 \% \mathrm{Cl}]$ & $p$ & \% explained ${ }^{3}$ & $\mathrm{~B}^{2}[95 \% \mathrm{Cl}]$ & $p$ & $\%$ explained ${ }^{3}$ \\
\hline Model 1: age and sex & $-2.04[-2.49 ;-1.58]$ & $<0.001$ & - & $-0.74[-1.19 ;-0.29]$ & 0.001 & - \\
\hline Model 2: model $1+$ ethnicity and wealth & $-1.48[-1.94 ;-1.03]$ & $<0.001$ & 27.5 & $-0.68[-1.14 ;-0.22]$ & 0.004 & 8.1 \\
\hline Model 3: model 2+ BMI & $-1.39[-1.84 ;-0.94]$ & $<0.001$ & 31.9 & $-0.68[-1.14 ;-0.22]$ & 0.004 & 8.1 \\
\hline Model 4: model $3+$ health behaviours ${ }^{4}$ & $-0.93[-1.42 ;-0.44]$ & $<0.001$ & 54.4 & $-0.50[-0.99 ;-0.02]$ & 0.044 & 32.4 \\
\hline Model 5: model $4+$ health status $^{5}$ & $-0.50[-0.99 ;-0.02]$ & 0.044 & 75.5 & $-0.44[-0.94 ; 0.05]$ & 0.078 & 40.5 \\
\hline Model 6: model $5+$ depressive symptoms & $-0.35[-0.84 ; 0.15]$ & 0.167 & 82.8 & $-0.44[-0.94 ; 0.06]$ & 0.084 & 40.5 \\
\hline
\end{tabular}

All figures are weighted for sampling probabilities and differential non-response.

${ }^{1}$ Prospective results are additionally adjusted for baseline handgrip strength.

${ }^{2} \mathrm{~B}$ values can be interpreted as the adjusted mean difference in handgrip strength in kilograms between the groups reporting good and poor eyesight.

${ }^{3}$ Percentage of age and sex-adjusted association between self-rated eyesight and handgrip strength explained by other variables included in the model.

${ }^{4}$ Health behaviours include physical activity, smoking status, alcohol intake.

${ }^{5}$ Health status includes limiting long-standing illness and disability.

$\mathrm{BMI}=$ body mass index; $\mathrm{Cl}=$ confidence interval. 